\title{
ON A FAMILY OF DENDRITES
}

\author{
JANUSZ J. CHARATONIK
}

(Received 25 June 2000)

\begin{abstract}
We study the open images of members of a countable family $\mathscr{F}$ of dendrites. We show that only two members of $\mathscr{F}$ are minimal and only one of them is unique minimal with respect to open mappings.
\end{abstract}

2000 Mathematics Subject Classification. 54E40, 54F15, 54F50.

1. Introduction. Let $\mathscr{S}$ be a family of topological spaces and $\mathbb{E}$ a class of mappings between members of $\mathscr{Y}$. Then $\mathscr{Y}$ can be quasi-ordered with respect to $\mathbb{F}$, writing for any $X, Y \in \mathscr{S}$

$$
\begin{gathered}
\left(Y \leq_{\mathbb{F}} X\right) \Longleftrightarrow(\text { there exists a surjection } f \in \mathbb{F} \text { of } X \text { onto } Y), \\
\left(X=_{\mathbb{F}} Y\right) \Longleftrightarrow\left(Y \leq_{\mathbb{F}} X \text { and } X \leq_{\mathbb{F}} Y\right) .
\end{gathered}
$$

A member $X_{0}$ of $\mathscr{Y}$ is said to be

- minimal in $\mathscr{S}$ with respect to $\mathbb{F}$ provided that, for each $Y$ in $\mathscr{S}$ the condition $Y \leq_{\mathbb{F}} X_{0}$ implies $Y=_{\mathbb{F}} X_{0}$;

- unique minimal in $\mathscr{S}$ with respect to $\mathbb{F}$ provided that for each $Y$ in $\mathscr{Y}$ if $Y \leq_{\mathbb{F}} X_{0}$ then $Y$ is homeomorphic to $X_{0}$.

Thus, in particular, all spaces in $\mathscr{S}$ which are homeomorphic to all its images under mappings belonging to $\mathbb{F}$ are unique minimal in $\mathscr{S}$ with respect to $\mathbb{F}$. (See $[5$, Chapter 3 , page 7] for more information.)

In this paper, we take as $\mathscr{S}$ the family $\mathscr{D}$ of dendrites (i.e., locally connected continua containing no simple closed curves) and as $\mathbb{E}$ the class $\mathbb{O}$ of open mappings (i.e., ones which map open subsets of the domain onto open subsets of the range). Various properties of the relation $\leq_{\mathbb{O}}$ on the family $\mathscr{D}$ are discussed in [5, Chapter 6, pages 2251]. Examples of dendrites which are homeomorphic to all its open images can be found, for example, in [2, Corollary, page 493 and the paragraph following it] and in [5, Theorem 6.45, page 30].

Answering a question in [5, Q2(O), page 51] (see also [3, Section 6, 2, page 245]) a dendrite $C$ is constructed in $[9$, Section 2] which is minimal with respect to $\mathbb{O}$ and which has two topologically distinct open images, thus is not unique minimal with respect to $\mathbb{O}$ (see [9, Proposition 3.5 $(\alpha)]$ ). The quoted paper contains also a construction of a countable family $\mathscr{F}$ of dendrites, with $C \in \mathscr{F}$. Since each member of $\mathscr{F}$ has a similar structure as the one of $C$, it is natural to ask about open mapping properties of other members of $\mathscr{F}$, especially properties which are related to the minimality of members of $\mathscr{F}$ with respect to the class $\mathbb{O}$. This is a subject of the present paper. 
All spaces considered in the paper are assumed to be metric and all mappings are continuous. A continuum means a compact connected space. Given a space $X$ and its subset $S$, we denote by cl $S$ the closure of $S$ and by int $S$ its interior in $X$. As usual $\mathbb{N}$ denotes the set of positive integers, and $\mathbb{R}$ stands for the space of real numbers.

We will use the notion of order of a point in the sense of Menger-Urysohn (cf. [7, Section 51, I, page 274]), and we denote by $\operatorname{ord}(p, X)$ the order of the space $X$ at a point $p \in X$. It is well known (cf. [7, Section 51, pages 274-307]) that the function ord takes its values from the set

$$
S=\left\{0,1,2, \ldots, n, \ldots, \omega, \aleph_{0}, 2^{\aleph_{0}}\right\} .
$$

Points of order 1 in a space $X$ are called end points of $X$; the set of all end points of $X$ is denoted by $E(X)$. Points of order 2 are called ordinary points of $X$. It is known that the set of all ordinary points is a dense subset of a dendrite. And for each $r \in$ $\left\{3,4, \ldots, \omega, \aleph_{0}, 2^{\aleph_{0}}\right\}$ points of order $r$ are called ramification points of $X$; the set of all ramification points is denoted by $R(X)$. It is known that for each dendrite $X$ the set $R(X)$ is at most countable, and that the points of order $\mathrm{N}_{0}$ and $2^{\mathrm{N}_{0}}$ do not occur in any dendrite.

A space $X$ is said to be universal in a class of spaces if it belongs to the class and it contains a homeomorphic copy of every element of that class.

2. The construction. It should be stressed that the construction below is modeled onto the one described in [9, Section 2], and also the proofs of the properties of the dendrites $D(r, s)$ are patterned after the corresponding ones presented in [9, Sections 2 and 3].

To construct the mentioned family $\mathscr{F}$ of dendrites, we fix some notation and terminology. For $n \in \mathbb{N}$ let $F_{n}$ denote the simple $n$-od, that is, a continuum homeomorphic to the cone over a (discrete) set of $n$ points. The vertex of the cone is called the vertex of $F_{n}$. In the Cartesian coordinates in the plane $\mathbb{R}^{2}$ put $v=(0,0)$, and for each $n \in \mathbb{N}$ let $e_{n}=\left(1 / n, 1 / n^{2}\right)$. Denoting by $p q$ the straight line segment in the plane with end points $p$ and $q$, define

$$
F_{\omega}=\bigcup\left\{v e_{n}: n \in \mathbb{N}\right\}
$$

The continua $F_{n}$ and $F_{\omega}$ are called fans of order $n$ and $\omega$, respectively. Any fan of order $n \in \mathbb{N}$ (thus having the set $E\left(F_{n}\right)$ of its end points finite) is also named a finite fan, and $F_{\omega}$ is also termed an infinite locally connected fan. Obviously fans $F_{n}$ and $F_{\omega}$ are dendrites.

An arc $p q$ with end points $p$ and $q$ in a continuum $X$ is called a free arc provided that $p q \backslash\{p, q\}$ is an open subset of $X$. If a free arc is not properly contained in another one, it is called a maximal free arc. Then three cases are possible: either both $p$ and $q$ are ramification points (and then it is called an interior free arc), or one of them is a ramification point and the other is an end point of $X$ (and then $p q$ is called an end free $\operatorname{arc}$ ), or finally both $p$ and $q$ are end points of $X$. Note that the third possibility holds only in a trivial case when $X=p q$. 
TheOREM 2.1. For every $r \in\left\{3,4, \ldots, \mathrm{N}_{0}\right\}$ and $s \in\left\{0,1,2, \ldots, \mathrm{N}_{0}\right\}$ there exists a dendrite $D(r, s)$ such that:

(2.1.1) each ramification point of $D(r, s)$ belongs to exactly $r$ interior free arcs in $D(r, s)$;

(2.1.2) each ramification point of $D(r, s)$ belongs to exactly $s$ end free arcs in $D(r, s)$;

(2.1.3) any two ramification points of $D(r, s)$ are contained in an arc in $D(r, s)$ containing only finitely many ramification points of $D(r, s)$.

Moreover, conditions (2.1.1), (2.1.2), and (2.1.3) determine the dendrite $D(r, s)$ up to a homeomorphism.

Proof. Let $X_{1}=F_{r}^{1} \cup F_{s}^{1}$ be the one-point union of the fans $F_{r}^{1}$ and $F_{s}^{1}$ such that $F_{r}^{1} \cap F_{s}^{1}=\{v\}$, where $v$ is the common vertex of the two fans. If $s=0$, we take $F_{s}=\{v\}$, and if $s=1$ or $s=2$ we understood $F_{s}^{1}$ as the union of one or two arcs, respectively, emanating from the point $v$ and disjoint out of this point. Thus $X_{1}$ is a fan with the vertex $v$, either finite or homeomorphic to $F_{\omega}$. In the set $E\left(X_{1}\right)$ we distinguish a subset $E_{1}=E\left(F_{r}^{1}\right)$.

Assume that a dendrite $X_{n}$ is defined for some $n \in \mathbb{N}$ and that in the set $E\left(X_{n}\right)$ of its end points a nonempty subset $E_{n}$ is distinguished. Consider the one point union $U=F_{r-1} \cup F_{S}$ where the vertices of the fans $F_{r-1}$ and $F_{S}$ are identified to a point $v(U)$. Then $X_{n+1}$ is obtained from $X_{n}$ by attaching to each end point $e \in E_{n} \subset X_{n}$ a properly diminished copy $U(e)=F_{r-1}^{n+1}(e) \cup F_{s}^{n+1}(e)$ of $U$ with the points $e \in X_{n}$ and $v(U(e)) \in U(e)$ identified, in such a way that $X_{n} \cap U(e)=\{e\}$, where $F_{r-1}^{n+1}(e)$ and $F_{s}^{n+1}(e)$ denote the corresponding copies of the fans $F_{r-1}$ and $F_{s}$, respectively. Thus $X_{n+1}$ is a dendrite by its definition. Further, define $E_{n+1}=\bigcup\left\{E\left(F_{r-1}(e)\right): e \in E_{n}\right\}$.

Note that $X_{n} \subset X_{n+1}$ for each $n \in \mathbb{N}$. We assume that the diameters of the components of $X_{n+1} \backslash X_{n}$ tend to 0 if $n$ tends to infinity. Let $f_{n}: X_{n+1} \rightarrow X_{n}$ be a monotone retraction. Thus $f_{n}$ shrinks each of the attached fans $U(e)$ back to its vertex $v(U(e))$ which is identified with the corresponding end point $e \in E_{n} \subset E\left(X_{n}\right)$.

Consider the inverse sequence $\left\{X_{n}, f_{n}: n \in \mathbb{N}\right\}$ of dendrites $X_{n}$ with monotone bonding mappings $f_{n}$, and define

$$
D(r, s)=\lim _{\{}\left\{X_{n}, f_{n}: n \in \mathbb{N}\right\} .
$$

By [8, Theorem 10.36, page 180 and Theorem 2.10, page 23] the defined inverse limit $D(r, s)$ is a dendrite which is homeomorphic to $\operatorname{cl}\left(\bigcup\left\{X_{n}: n \in \mathbb{N}\right\}\right)$. Neglecting the homeomorphism we can simply write

$$
D(r, s)=\operatorname{cl}\left(\bigcup\left\{X_{n}: n \in \mathbb{N}\right\}\right) .
$$

It is evident from the construction that $D(r, s)$ has properties (2.1.1), (2.1.2), and (2.1.3). In [9, Proposition 3.3] it is proved that these properties uniquely determine $D(r, s)$. The proof is then complete.

Finally we put

$$
\mathscr{F}=\left\{D(r, s): r \in\left\{3,4, \ldots, \aleph_{0}\right\}, s \in\left\{0,1,2, \ldots, \aleph_{0}\right\}\right\} .
$$


Properties (2.1.1) and (2.1.2) imply the following.

STATEMENT 2.2. The dendrite $D(r, s)$ is composed exclusively of points of order 1 , 2 , and $r+s$, with a convention that, in the case when one of $r$ or $s$ is $\kappa_{0}$, points of order $r+s$ are understood as ones of order $\omega$.

The next statement is a consequence of property (2.1.3).

STATEMENT 2.3. Let an integer $r \geq 3$ and $s \in\left\{0,1,2, \ldots, \aleph_{0}\right\}$ be fixed. If $\left\{p_{m}: m \in \mathbb{N}\right\}$ is a convergent sequence of distinct ramification points of $D(r, s)$, then $\lim p_{m}$ is an end point.

As a consequence of (2.3) and Statement 2.3, we get the following inclusion.

$$
W=\operatorname{cl}\left(\bigcup\left\{X_{n}: n \in \mathbb{N}\right\}\right) \backslash \bigcup\left\{X_{n}: n \in \mathbb{N}\right\} \subset E(D(r, s)) .
$$

The next inclusion is obvious.

$$
\begin{gathered}
D\left(r_{1}, s_{1}\right) \subset D\left(r_{2}, s_{2}\right) \quad \text { for every } r_{1}, r_{2} \in\left\{3,4, \ldots, \aleph_{0}\right\}, \\
s_{1}, s_{2} \in\left\{0,1,2, \ldots, \aleph_{0}\right\} \quad \text { with } r_{1} \leq r_{2}, s_{1} \leq s_{2} .
\end{gathered}
$$

In particular, we have the following:

$$
\begin{aligned}
& D(r, 0) \subset D(r, 1) \subset D(r, 2) \subset \cdots \subset D\left(r, \aleph_{0}\right) \text { for each } r \in\left\{3,4, \ldots, \aleph_{0}\right\}, \\
& D(3, s) \subset D(4, s) \subset D(5, s) \subset \cdots \subset D\left(\aleph_{0}, s\right) \text { for each } s \in\left\{0,1,2, \ldots, \aleph_{0}\right\} .
\end{aligned}
$$

Note that $D\left(\kappa_{0}, 1\right)$ is $C_{\omega}^{1}$ of [9].

For each integer $n \geq 3$, a dendrite $G^{n}$ is constructed in [1, Chapter 4] which is universal in the class of all dendrites with a closed set of end points and of orders of their ramification points not greater than $n$ (see [1, Theorems 4.1 and 4.2]). Comparing the two constructions, namely, of $D(r, s)$ and of $G^{n}$, it is evident that

$$
D(r, 0) \text { is homeomorphic to } G^{r} \text { for each integer } r \geq 3 \text {, }
$$

whence it follows from (2.7) that for every $r \in\{3,4, \ldots\}$ and $s \in\left\{0,1,2, \ldots, \mathrm{N}_{0}\right\}$ the dendrite $G^{r}$ is contained in $D(r, s)$ even in such a way that $E\left(G^{r}\right) \subset E(D(r, s))$.

The next result follows from [1, Theorem 3.3] which gives a characterization of dendrites with a closed set of end points. But it is also a direct consequence of the definition of $D(r, s)$.

Proposition 2.4. If $r \in\{3,4,5, \ldots\}$ and $s \in\{0,1,2, \ldots\}$, then the dendrite $D(r, s)$ has a closed set of end points.

Therefore Proposition 2.4, (2.9), and the above mentioned universality of dendrites $G^{n}$ imply the following corollary.

Corollary 2.5. If $r \in\{3,4,5, \ldots\}$ and $s \in\{0,1,2, \ldots\}$, then $D(r, s)$ can be embedded in $D(r+s, 0)$. 
3. Open images. In this section, we intend to study open images of members of the family $\mathscr{F}$. We start with recalling some theorems which are useful tools for the study of open mapping properties of some continua.

Proposition 3.1. Let a mapping $f: X \rightarrow Y$ be a nonconstant open surjection between continua. Then

(3.1.1) the order of a point is not increased, that is, ord $(f(x), Y) \leq \operatorname{ord}(x, X)$; in particular $f(E(X)) \subset E(Y)$;

(3.1.2) if $X$ is an arc, or a dendrite, or the infinite locally connected fan $F_{\omega}$, then so is $Y$;

(3.1.3) if $X$ is a dendrite, then

(a) $f^{-1}(y)$ is finite for each $y \in Y \backslash E(Y)$;

(b) $f^{-1}(E(Y)) \backslash E(X)$ is finite;

(c) if $\operatorname{ord}(x, X)=\omega$, then $\operatorname{ord}(f(x), Y)=\omega$;

(d) the image under $f$ of a free arc in $X$ is a free arc in $Y$.

Proof. The proof of (3.1.1) follows from [10, Chapter 8, (7.31), page 147].

(3.1.2): for an arc and a dendrite see [10, Chapter 10, (1.3), page 184], [10, Chapter 8 , (7.7), page 148 , and Chapter 10 , page 185$]$; compare [5, $(6.1)$, page 22]; for $F_{\omega}$ see [4, Proposition 9.4, page 42].

(3.1.3): see [5, Propositions 6.16, page 25, 6.5 and Corollary 6.7, page 23].

Using the above facts concerning open mappings, the following proposition is shown in [9, Proposition 3.5 $(\alpha)$ ].

Proposition 3.2. Each open image of the dendrite $D\left(\mathrm{~N}_{0}, 1\right)$ is homeomorphic either to $D\left(\kappa_{0}, 1\right)$ or to the one-point union $U$ of $D\left(\kappa_{0}, 1\right)$ with an end free arc $p q$, where $p \in R\left(D\left(\aleph_{0}, 1\right)\right)$.

Recall that an open mapping of $D\left(\kappa_{0}, 1\right)$ onto the union $U$ is obtained as follows: locate $D\left(\kappa_{0}, 1\right)$ in the plane in such a way that

(1) all free arcs in $D\left(\kappa_{0}, 1\right)$ are straight line segments, and

(2) $D\left(\aleph_{0}, 1\right)$ is symmetric with respect to a straight line $L$ which is perpendicular to an interior free segment $S$ and passes through its mid point $m$ so that $D\left(\kappa_{0}, 1\right) \cap L=\{m\}$ (see [9, Figure 3]).

Then the mentioned open mapping is the identity on one half of $D\left(\mathrm{~N}_{0}, 1\right)$ (lying on one half-plane determined by $L$ ) and it is the symmetry on the other; equivalently, if $\sim$ denotes the symmetry with respect to $L$, then $U$ is homeomorphic to $D\left(\kappa_{0}, 1\right) / \sim$, and $p q$ is homeomorphic to $S / \sim$.

Exactly the same arguments as in the proof of [9, Proposition 3.5] can be applied to show the next two propositions. In particular, observe that if the above recalled open mapping is applied to $D\left(\aleph_{0}, \aleph_{0}\right)$, then the resulting union $U$ is homeomorphic to $D\left(\aleph_{0}, \aleph_{0}\right)$. The details are left to the reader.

Proposition 3.3. Each open image of the dendrite $D\left(\mathrm{~N}_{0}, 0\right)$ is homeomorphic either to $D\left(\aleph_{0}, 0\right)$ or to the one-point union $U$ of $D\left(\kappa_{0}, 0\right)$ with an end free arc $p q$, where $p \in R\left(D\left(\kappa_{0}, 0\right)\right)$. 
Proposition 3.4. Each open image of the dendrite $D\left(\mathrm{~N}_{0}, \mathrm{~N}_{0}\right)$ is homeomorphic to $D\left(\aleph_{0}, \aleph_{0}\right)$.

REMARK 3.5. According to (3.1.2), an arc and $F_{\omega}$ are examples of dendrites homeomorphic to their open images. Recall that such dendrites are said to be unique minimal elements of the class $\mathscr{D}_{\leq_{\mathbb{O}}}$ (see [5, Chapter 3, pages 7-8]). Among all universal dendrites $D_{S}$ with $S \subset\{3,4, \ldots, \omega\}$, only $D_{3}, D_{\omega}$ and $D_{\{3, \omega\}}$ have this property (see [2, Corollary, page 493] and [6, Corollary 6.10, page 232]). An uncountable family of some dendrites such that each member of the family is homeomorphic to any of its open images is constructed in [5, Theorem 6.45, page 30]. However, the internal structure of all dendrites having the considered property is not known (see [5, Chapter 7 , problem Q1(O), page 51]). Proposition 3.4 gives a new example of a dendrite which is a unique minimal element of the class $\mathscr{D}_{\leq_{\mathbb{O}}}$.

As it is shown in [9, Proposition 3.5 $(\gamma)$ ], each open image of $D\left(\kappa_{0}, 1\right)$ can by mapped onto $D\left(\aleph_{0}, 1\right)$ under an open mapping, that is, $D\left(\kappa_{0}, 1\right)$ is a minimal (but not unique minimal, according to Proposition 3.2) element of the class $\mathscr{D}_{\leq_{\mathbb{O}}}$. Thus [5, Chapter 7 , problem Q1(O), page 51] has a negative answer (this is the main result of [9]). Note that it is not the case for $D\left(\aleph_{0}, 0\right)$ because (by (3.1.1) above) the union $U$ of Proposition 3.3 cannot be openly mapped onto $D\left(\aleph_{0}, 0\right)$. For further results in this direction see below.

Propositions 3.2, 3.3, and 3.4 describe open images of $D\left(\aleph_{0}, s\right)$ for $s \in\left\{0,1, \aleph_{0}\right\}$. For $s \in\{2,3, \ldots\}$ some open images of $D\left(\kappa_{0}, s\right)$ can be obtained in the following way. Fix any nonempty subset $P \subset R\left(D\left(\kappa_{0}, s\right)\right)$. For any ramification point $p \in P$ let $F_{S}(p) \subset$ $D\left(\kappa_{0}, s\right)$ be the union of $s$ end free $\operatorname{arcs} p e_{1}^{p}, p e s_{2}^{p}, \ldots, p e_{s}^{p}$, every two of which have the singleton $\{p\}$ in common only. Further, for a fixed $t \in\{1,2, \ldots, s\}$ let $F_{t}(p) \subset F_{s}(p)$ be the union of $t$ end free $\operatorname{arcs} p e_{i_{1}}^{p}, p e_{i_{2}}^{p}, \ldots, p e_{i_{t}}^{p}$. Then there is an open surjective mapping $f^{(p)}: F_{S}(p) \rightarrow F_{t}(p)$ which is a homeomorphism on each free arc $p e_{j}^{p}$ for each $j \in\{1,2, \ldots, s\}$ with $f^{(p)}(p)=p$ and $f^{(p)}\left(e_{j}^{p}\right)=e_{i_{j}}^{p}$ for some $i_{j} \in\left\{i_{1}, i_{2}, \ldots, i_{t}\right\}$. Then the mapping $f: D\left(\aleph_{0}, s\right) \rightarrow Y \subset D\left(\aleph_{0}, s\right)$ such that $f \mid F_{s}(p)=f^{(p)}$ for each $p \in$ $P$ and defined as a homeomorphism otherwise is obviously open. In particular, if $P=R\left(D\left(\kappa_{0}, s\right)\right)$ and if $t \in\{1,2, \ldots, s\}$ is the same fixed number for all ramification points $p$, then $Y=D\left(\kappa_{0}, t\right)$, and the following proposition is obtained.

Proposition 3.6. For each $s \in\{2,3, \ldots\}$ and for each $t \in\{1,2, \ldots, s\}$ there is an open mapping of $D\left(\mathrm{\kappa}_{0}, s\right)$ onto $D\left(\mathrm{\aleph}_{0}, t\right)$.

Taking $t=1$ in the above construction we conclude from Proposition 3.2 that for each $s \in\{2,3, \ldots\}$ there is no open mapping from $D\left(\kappa_{0}, 1\right)$ onto $D\left(\kappa_{0}, s\right)$. Therefore the next result follows.

Proposition 3.7. For each $s \in\{2,3, \ldots\}$ no dendrite $D\left(\kappa_{0}, s\right)$ is minimal in the class $\mathscr{D}_{\leq \mathbb{O}}$.

We now consider open images of other members of $\mathscr{F}$, namely of dendrites $D(r, s)$ for $r \in\{3,4, \ldots\}$ and $s \in\left\{0,1,2, \ldots, \aleph_{0}\right\}$. To see that no one of them is minimal in the class $\mathscr{D}_{\leq_{\mathbb{O}}}$ we need some facts about the structure of the set of end points of $D(r, s)$. To this aim represent $D(r, s)$ as in (2.2) and observe that if $r \neq \kappa_{0}$, then the 
set $\left(X_{n+1} \backslash X_{n}\right) \cap R(D(r, s))$ is finite. Putting

$$
R_{1}=\{v\}, \quad R_{n+1}=\left(X_{n+1} \backslash X_{n}\right) \cap R(D(r, s)) \quad \forall n \in \mathbb{N},
$$

we see that $R(D(r, s))=\bigcup\left\{R_{n}: n \in \mathbb{N}\right\}$, and that the sets $R_{n}$ are mutually disjoint. For each point $q \in R_{n}$ let $F_{s}^{n}(q)$ denote, as previously, the union of $s$ end free arcs in $D(r, s)$ every two of which have the point $q$ in common only. Since according to (2.5) the remainder $W$ consists of end points of $D(r, s)$ only, we have

$$
E(D(r, s))=W \cup\left(\bigcup\left\{\bigcup\left\{E\left(F_{s}^{n}(q)\right): q \in R_{n}\right\}: n \in \mathbb{N}\right\}\right) .
$$

Observe that, simply by the construction, each point of $E\left(F_{s}^{n}(q)\right)$ is an isolated point of $E(D(r, s))$. Further, since $G^{r}$ is homeomorphic to $D(r, 0) \subset D(r, s)$ by (2.7) and (2.9) and since $E\left(G^{r}\right)$ is homeomorphic to the Cantor set according to [1, Theorem 4.1], it follows again from (2.9) that

$$
W \text { is homeomorphic to the Cantor set. }
$$

Note further that if $K$ is a component of the set

$$
S=D(r, s) \backslash\left(W \cup \bigcup\left\{\bigcup\left\{F_{s}^{n}(q): q \in R_{n}\right\}: n \in \mathbb{N}\right\}\right),
$$

then

$$
\text { there is } n \in \mathbb{N} \text { such that } K \subset X_{n} \backslash R_{n} \text {, }
$$

$\operatorname{cl}(K)$ is an interior free arc of $D(r, s)$ with one end point in $R_{n}$ and the other in $R_{n+1}$.

Therefore $D(r, s)$ can be written as the following union:

$$
\begin{aligned}
D(r, s) & =W \cup(\bigcup\{K: K \text { is a component of } S\}) \\
& \cup\left(\bigcup\left\{\bigcup\left\{F_{s}^{n}(q): q \in R_{n}\right\}: n \in \mathbb{N}\right\}\right) .
\end{aligned}
$$

Now we are ready to show the next result.

EXAMPLE 3.8. For every $r \in\{3,4, \ldots\}$ and $s \in\left\{0,1,2, \ldots, \aleph_{0}\right\}$ there are a subdendrite $Y \subset D(r, s)$ and an open retraction $g: D(r, s) \rightarrow Y$ such that $D(r, s)$ is not an open image of $Y$.

Proof. Fix $r$ and $s$ as assumed. Let $D(r, s)$ be defined as the inverse limit by (2.2) and let, as previously, $v$ be the only ramification point of the fan $X_{1}$. For each $n \in \mathbb{N}$ choose a ramification point $p_{n} \in R_{n} \subset D(r, s)$. Thus $p_{1}=v$ and $p_{n} \in v p_{n+1}$ for each $n \in \mathbb{N}$. Then the sequence $\left\{p_{n}\right\}$ is convergent, and its limit $e_{0}=\lim p_{n}$ is, according to Statement 2.3, an end point of $D(r, s)$ lying in the set $W$. Thus all points $p_{n}$ lie in the arc $v e_{0}$, and if < is the natural ordering of $v e_{0}$ from $v$ to $e_{0}$, then

$$
v=p_{1}<p_{2}<\cdots<p_{n}<p_{n+1}<\cdots<e_{0} .
$$

Further, for each $n \in \mathbb{N}$ take $F_{s}^{n}\left(p_{n}\right) \subset X_{n} \subset D(r, s)$ and note that $v e_{0} \cap F_{s}^{n}\left(p_{n}\right)=\left\{p_{n}\right\}$. Put

$$
Y=v e_{0} \cup \bigcup\left\{F_{s}^{n}\left(p_{n}\right): n \in \mathbb{N}\right\} \subset D(r, s) .
$$


Define a mapping $g: D(r, s) \rightarrow Y$ such that

- $g \mid Y: Y \rightarrow Y$ is the identity;

- for each component $K=a b$ of $S$ as in (3.4), if $a \in R_{n}$ and $b \in R_{n+1}$, where $n$ is determined by (3.5), the restriction $g \mid K: K \rightarrow p_{n} p_{n+1} \subset v e_{0}$ is a homeomorphism such that $g(a)=p_{n}$ and $g(b)=p_{n+1}$;

- for each $F_{s}^{n}(q)$ with $q \in R_{n}$ for some $n \in \mathbb{N}$ the restriction $g \mid F_{s}^{n}(q): F_{s}^{n}(q) \rightarrow$ $F_{s}^{n}\left(p_{n}\right)$ is a homeomorphism;

- $g(W)=\left\{e_{0}\right\}$.

By (3.7) the mapping $g$ is well defined. It can be verified that $g$ is the needed open retraction.

To see that $Y$ cannot be openly mapped onto $D(r, s)$, it is enough to observe that the set $E(Y)$ is countable, while $E(D(r, s))$ is not countable by (2.9). Hence the conclusion follows from (3.1.1) and (3.1.3)(b).

COROLlaRY 3.9. For every $r \in\{3,4, \ldots\}$ and $s \in\left\{0,1,2, \ldots, \aleph_{0}\right\}$ the dendrite $D(r, s)$ is not minimal in the class $\mathscr{D}_{\leq_{\mathbb{O}}}$.

The above results can be summarized in the following theorem.

THEOREM 3.10. There are only two minimal elements of the class $\mathscr{D}_{\leq_{\mathbb{O}}}$ among all members $D(r, s)$ of $\mathscr{F}$ for $r \in\left\{3,4, \ldots, \aleph_{0}\right\}$ and $s \in\left\{0,1,2, \ldots, \aleph_{0}\right\}$, namely, $D\left(\kappa_{0}, 1\right)$ and $D\left(\mathrm{\kappa}_{0}, \mathrm{\aleph}_{0}\right)$. Only one of them, namely, $D\left(\mathrm{\kappa}_{0}, \mathrm{\aleph}_{0}\right)$, is unique minimal.

\section{REFERENCES}

[1] D. Arévalo, W. J. Charatonik, P. P. Covarrubias, and L. Simón, Dendrites with a closed set of end points, Topology Appl. 115 (2001), no. 1, 1-17. CMP 1840729.

[2] J. J. Charatonik, Open mappings of universal dendrites, Bull. Acad. Polon. Sci. Sér. Sci. Math. 28 (1980), no. 9-10, 489-494. MR 84a:54069. Zbl 469.54019.

[3] J. J. Charatonik and W. J. Charatonik, Dendrites, 30th National Congress of the Mexican Mathematical Society (Spanish) (Aguascalientes, 1997), Soc. Mat. Mexicana, Mexico, 1998, pp. 227-253. MR 2001f:54038. Zbl 01612606.

[4] J. J. Charatonik, W. J. Charatonik, and S. Miklos, Confluent mappings of fans, Dissertationes Math. (Rozprawy Mat.) 301 (1990), 1-86. MR 91h:54056. Zbl 776.54025.

[5] J. J. Charatonik, W. J. Charatonik, and J. R. Prajs, Mapping hierarchy for dendrites, Dissertationes Math. (Rozprawy Mat.) 333 (1994), 1-52. MR 96b:54021. Zbl 822.54009.

[6] W. J. Charatonik and A. Dilks, On self-homeomorphic spaces, Topology Appl. 55 (1994), no. 3, 215-238. MR 95a:54054. Zbl 788.54040.

[7] K. Kuratowski, Topology. Vol. II, Academic Press, New York, 1968. MR 41 \#4467.

[8] S. B. Nadler, Jr., Continuum Theory, Marcel Dekker, New York, 1992. MR 93m:54002. Zbl 757.54009.

[9] D. Opěla, P. Pyrih, and R. Šámal, An example of openly minimal dendrite, Questions Answers Gen. Topology 18 (2000), no. 2, 229-236. CMP 1786302.

[10] G. T. Whyburn, Analytic Topology, American Mathematical Society, New York, 1942. MR 4,86b.

Janusz J. Charatonik: Mathematical institute, University of Wroceaw, Pl. GRUNWALDZKI 2/4, 50-384 WrocŁaW, POLAND

Current address: InSTITUTO DE MATEMÁTICAS, UNAM, CiRCUITO EXTERIOR, CIUDAD UNIVERSITARIA, 04510 MeXICO, D.F., MeXICO

E-mail address: jjc@hera.math.uni.wroc.p1; jjc@matem.unam.mx 


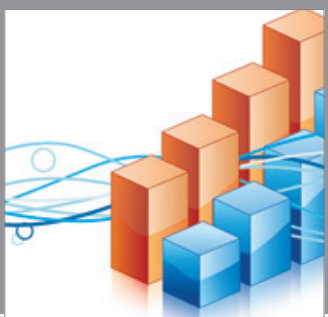

Advances in

Operations Research

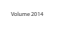

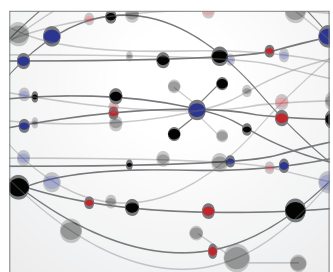

\section{The Scientific} World Journal
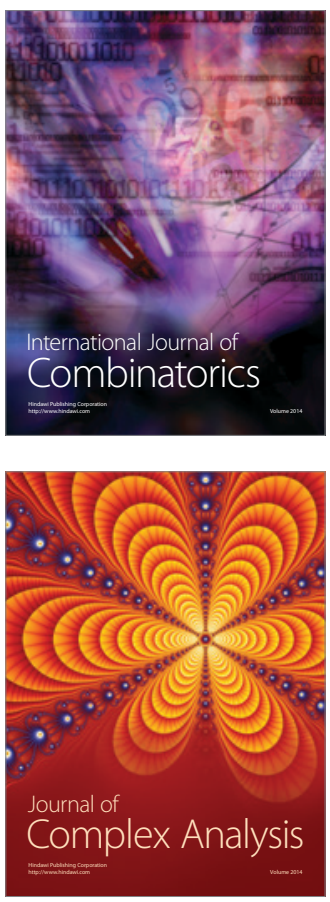

International Journal of

Mathematics and

Mathematical

Sciences
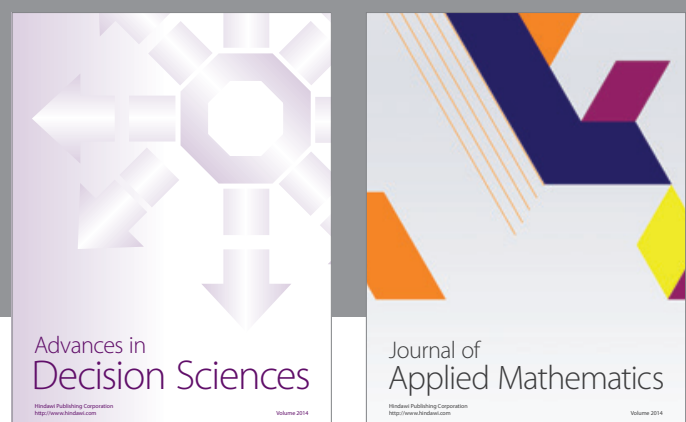

Journal of

Applied Mathematics
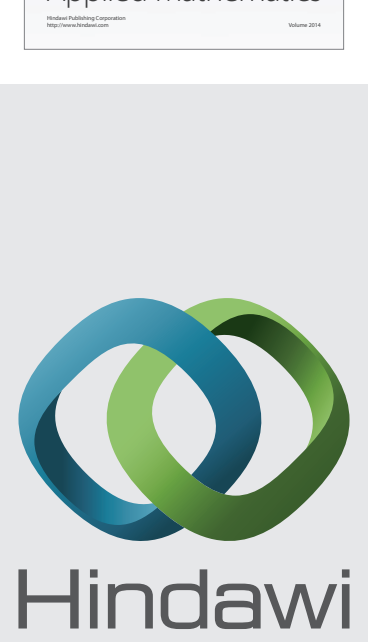

Submit your manuscripts at http://www.hindawi.com
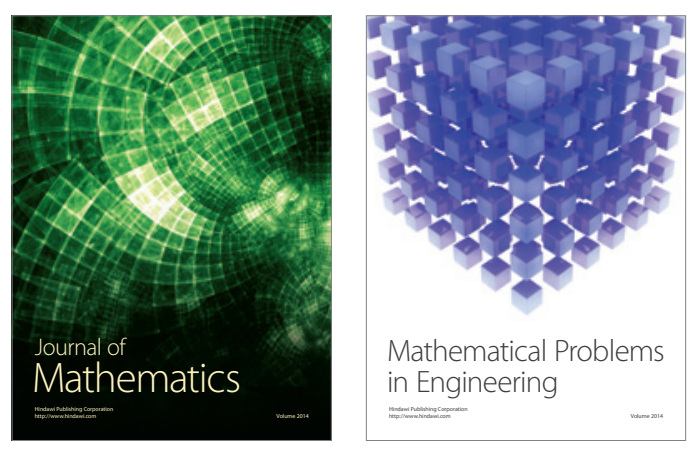

Mathematical Problems in Engineering
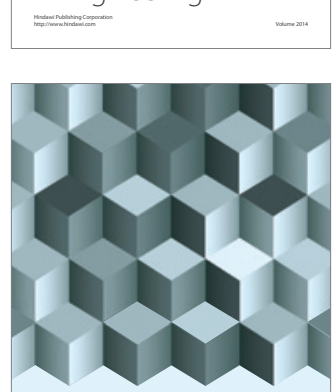

Journal of

Function Spaces
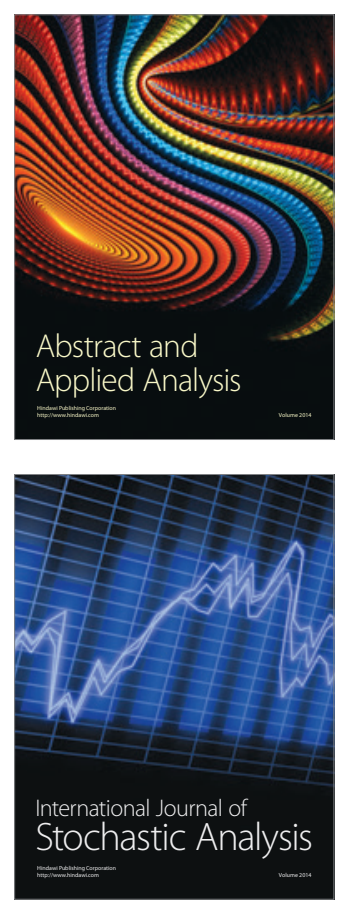

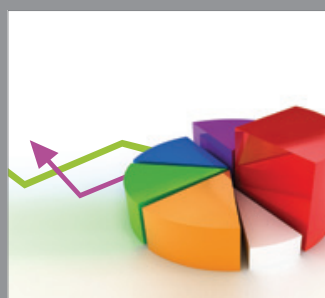

ournal of

Probability and Statistics

Promensencen
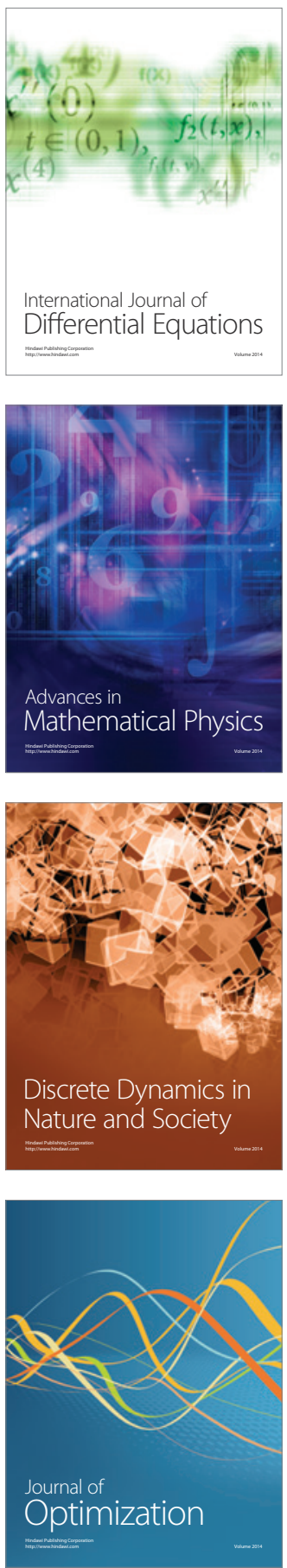